\title{
miR-25-3p promotes glioma cell proliferation and migration by targeting FBXW7 and DKK3
}

\author{
GANG PENG, CHENXING YANG, YI LIU and CHENFU SHEN \\ Department of Neurosurgery, Xiangya Hospital of Central South University, Changsha, Hunan 410008, P.R. China
}

Received August 6, 2018; Accepted April 5, 2019

DOI: $10.3892 /$ etm.2019.7583

\begin{abstract}
MicroRNAs (miRs) serve important roles in glioma. However, the underlying molecular mechanism of miR-25 in glioma progression remains largely unknown; therefore, it was investigated in the present study. RT-qPCR analysis revealed that miR-25 expression levels were markedly increased in human glioma tissue and glioma cell lines compared with normal brain tissues and normal human astrocytes, respectively. miR-25 upregulation exhibited an association with glioma progression, and the knockdown of miR-25 significantly inhibited glioma cell proliferation and migration. F-box and WD repeat domain containing 7 (FBXW7) and dickkopf WNT signaling pathway inhibitor 3 (DKK3) were identified as target genes of miR-25. FBXW7 and DKK3 expression levels were significantly downregulated in glioma tissue samples compared with normal brain tissue, and their expression levels were negatively regulated by miR-25 expression in glioma cells. Furthermore, inhibition of FBXW7 and DKK3 expression suppressed the miR-25-induced effects on glioma cell proliferation and migration. The findings of the present study suggest that miR-25 may promote glioma cell proliferation and migration by inhibiting the expression of FBXW7 and DKK3. Therefore, miR-25 may serve as a promising molecular target for the treatment of glioma.
\end{abstract}

\section{Introduction}

Glioma is one of the most common types of malignant brain tumour, with a morbidity rate of 5/10,000 each year $(1,2)$. During glioma progression, a series of molecular events result in the expression of genes associated with tumour cell proliferation and motility $(3,4)$. Investigating the underlying molecular mechanisms in glioma cell growth and progression

Correspondence to: Professor Chenfu Shen, Department of Neurosurgery, Xiangya Hospital of Central South University, 87 Xiangya Road, Changsha, Hunan 410008, P.R. China

E-mail: scf680524@126.com

Key words: glioma, microRNA, F-box and WD repeat domain containing 7, dickkopf Wnt signaling pathway inhibitor 3, proliferation, migration by gene expression profiling may facilitate the identification of potentially novel biomarkers and therapeutic targets.

MicroRNAs (miRs), which are small endogenous noncoding RNAs comprising 22-25 nucleotides, regulate gene expression at the transcriptional and/or post-transcriptional level by binding to the 3'untranslated region (UTR) of their target mRNA $(5,6)$. By repressing protein translation or promoting mRNA degradation, miRs play important roles in multiple cellular processes, including cell proliferation, survival, apoptosis, differentiation and motility (6-8). Several studies have demonstrated that miRs are deregulated in glioma, and some miRs have been found to play promoting or suppressive roles in different types of cancer $(9,10)$. For instance, miR-365 is downregulated in glioma and inhibits glioma cell proliferation, migration and invasion via targeting phosphoinositide-3-kinase (PI3K) regulatory subunit 3 (9), while miR-93 is upregulated in glioma and promotes glioma cell proliferation via activation of the PI3K/Akt signalling pathway (11). Among the glioma-related miRs, miR-25 is upregulated in several types of human cancer and often serves an oncogenic role $(12,13)$. For instance, miR-25 promotes the proliferation of triple-negative breast cancer by targeting BTG anti-proliferation factor 2 (12). Furthermore, exosome-derived miR-25 stimulates the malignant progression of liposarcoma (13). Zhang et al (14) reported that miR-25 promoted glioma cell proliferation by targeting cyclin dependent kinase inhibitor 1C, while Peng et al (15) demonstrated that miR-25 promoted glioblastoma cell proliferation and invasion by directly targeting neurofilament light. However, the clinical significance of miR-25 expression in glioma as well as the underlying molecular mechanism of miR-25 in glioma cell proliferation and migration remains unknown.

F-box and WD repeat domain containing 7 (FBXW7), a substrate adaptor for an E3 Skp1-Cul1-F-box ubiquitin ligase complex, negatively regulates the abundance of several oncoproteins (16). Studies have demonstrated that miRs may serve promoting roles in several types of human cancer via targeting FBXW7 (17,18). For instance, miR-92a is upregulated in cervical cancer and promotes cell proliferation and invasion by targeting FBXW7 (17). In addition, FBW7 inhibits malignancy and enhances temozolomide sensitivity in glioblastoma cells (18). However, the relationship between miR-25 and FBXW7 in glioma has not previously been identified.

Dickkopf Wnt signaling pathway inhibitor 3 (DKK3), a member of the Dickkopf family, interacts with and suppresses 
the Wnt signalling pathway and tumourigenesis $(19,20)$. DKK3 is downregulated in several types of human cancer and is a tumour suppressor (20). A previous study demonstrated that DKK3 induced glioma cell death (21). However, the relationship between miR-25 and DKK3 in glioma has not previously been examined.

In the present study, upregulation of miR-25 expression in glioma was associated with poor survival in patients with glioma. In addition, FBXW7 and DKK3 were identified as potential targets of miR-25 in glioma cells. Furthermore, miR-25 promoted glioma cell survival, proliferation and migration via targeting FBXW7 and DKK3.

\section{Materials and methods}

Tissue samples. A total of 60 primary glioma tissue and 10 normal brain tissue samples were collected from patients undergoing surgical resection at the Department of Neurosurgery of Xiangya Hospital (Changsha, China) between March 2010 and May 2014. The glioma tissue was obtained from 60 patients with glioma (female, $n=25$; male, $n=35$; age range, 32-66 years; mean age, 55.1 years), and normal tissue adjacent to the tumour was obtained from 10 patients (female, $n=4$; male, $n=6$; age range, 40-65 years; mean age, 53.6 years). None of the patients had received chemotherapy or radiotherapy prior to surgical resection. The WHO stage was determined by pathologists (22). All of the tissue samples collected were immediately snap-frozen in liquid nitrogen and stored at $-80^{\circ} \mathrm{C}$. According to the Helsinki Declaration, all participants or their families were well informed of the study details and written informed consent was obtained prior to the study. The current study was approved by the Ethics Committee of Xiangya Hospital of Central South University (Changsha, China).

Cell culture. Normal human astrocytes (NHAs) were obtained from Lonza Group Ltd. The human glioma cell lines U-373MG Uppsala, U-87MG Uppsala, U251 and T98G were obtained from the Cell Bank of Type Culture Collection of Chinese Academy of Sciences (Shanghai, China). Cells were cultured in Dulbecco's modified Eagle's medium (DMEM; Gibco; Thermo Fisher Scientific, Inc.) supplemented with 10\% FBS (Thermo Fisher Scientific, Inc.), 1\% penicillin and streptomycin (Thermo Fisher Scientific, Inc.), and 1\% glutamine (Thermo Fisher Scientific, Inc.) at $37^{\circ} \mathrm{C}$ in a humidified atmosphere containing $5 \% \mathrm{CO}_{2}$.

Cell transfection. U251 and T98G cells in the logarithmic phase were seeded at a density of $5 \times 10^{5}$ cells/well in a six-well plate for $24 \mathrm{~h}$. The cells were then transfected with $100 \mathrm{nM}$ negative control (NC) inhibitor (cat. no. 4464076; Thermo Fisher Scientific, Inc.), $100 \mathrm{nM}$ miR-25 inhibitor (cat. no. 4464084; Thermo Fisher Scientific, Inc.), $100 \mathrm{nM}$ miR-NC (cat. no. 4464058; Thermo Fisher Scientific, Inc.) or $100 \mathrm{nM}$ miR-25 mimics (cat. no. 4464066; Thermo Fisher Scientific, Inc.); or co-transfected with $100 \mathrm{nM}$ miR-25 inhibitor and $100 \mathrm{nM} \mathrm{NC}$ siRNA (cat. no. 4390843; Thermo Fisher Scientific, Inc.; group termed miR-25 in + siNC), $100 \mathrm{nM}$ miR-25 inhibitor and $100 \mathrm{nM}$ FBXW7 siRNA (cat. no. HSS124318; Thermo Fisher Scientific, Inc.; group termed miR-25 in + siFBXW7) or $100 \mathrm{nM}$ miR-25 inhibitor and 100 nM DKK3 siRNA (cat. no. HSS146899; Thermo Fisher Scientific, Inc.; group termed miR-25 in + siDKK3) using Lipofectamine ${ }^{\circledR} 2000$ (Thermo Fisher Scientific, Inc.), according to the manufacturer's protocol. Following transfection for $48 \mathrm{~h}$, transfected cells were collected and used in subsequent experimentation.

Reverse transcription-quantitative polymerase chain reaction $(R T-q P C R)$. Total RNA was extracted from tissue samples or cells using TRIzol $^{\circledR}$ reagent (Invitrogen; Thermo Fisher Scientific, Inc.). Total RNA was subsequently reverse transcribed into cDNA using the miScript Reverse Transcription kit (Qiagen, Inc.), according to the manufacturer's protocol using the following conditions: $37^{\circ} \mathrm{C}$ for $60 \mathrm{~min}$ and $95^{\circ} \mathrm{C}$ for 5 min, followed by storage at $4^{\circ} \mathrm{C}$. qPCR was subsequently performed using the miScript SYBR ${ }^{\circledR}$ Green PCR kit (Qiagen, Inc.) on a Roche LightCycler 480 Real-Time PCR system (Roche Diagnostics). The following thermocycling conditions were used for qPCR: Initial denaturation at $95^{\circ} \mathrm{C}$ for $1 \mathrm{~min}$; 40 cycles of $95^{\circ} \mathrm{C}$ for $15 \mathrm{sec}, 55^{\circ} \mathrm{C}$ for $30 \mathrm{sec}$ and $72^{\circ} \mathrm{C}$ for $30 \mathrm{sec}$. The mRNA expression levels were quantified using the $2^{-\Delta \Delta C q}$ method (23). The following primer pairs were used for the qPCR: miR-25 forward, 5'-CATTGCACTTGTCTC GGTCTGA-3' and reverse, 5'-GCTGTCAACGATACGCTA CGTAACG-3'; U6 forward, 5'-CTCGCTTCGGCAGCACA-3', and reverse, 5'-AACGCTTCACGAATTTGCGT-3'; DKK3 forward, 5'-AGGACACGCAGCACAAATTG-3' and reverse, 5'-CCAGTCTGGTTGTTGGTTATCTT-3'; FBXW7 forward, 5'-GGCCAAAATGATTCCCAGCAA-3' and reverse, 5'-ACT GGAGTTCGTGACACTGTTA-3'; and GAPDH forward, 5'-CTGGGCTACACTGAGCACC-3' and reverse, 5'-AAG TGGTCGTTGAGGGCAATG-3'.

Cell Counting kit-8 (CCK-8) assay. CCK-8 assays were performed to assess cell proliferation. Transfected cells were seeded into 96 -well plates at a density of $5 \times 10^{3}$ cells/well. Following incubation at $37^{\circ} \mathrm{C}$ for $0,24,48$ and $72 \mathrm{~h}, 10 \mu \mathrm{l}$ CCK-8 reagent (Beyotime Institute of Biotechnology) was add to each well. Cells were further incubated $37^{\circ} \mathrm{C}$ for $30 \mathrm{~min}$ and the absorbance was measured at a wavelength of $490 \mathrm{nM}$ using a microplate reader.

Wound-healing assay. Cell migration was examined using wound-healing assays. Transfected U251 and T98G cells were seeded into 12 -well plates at a density of $5 \times 10^{5}$ cells/well. Following incubation at $37^{\circ} \mathrm{C}$ for $24 \mathrm{~h}$, cells were scratched with a sterile 200- $\mu 1$ pipette tip and PBS was used to wash away any detached cells. Cell migration was observed following culture at $37^{\circ} \mathrm{C}$ for 0 and $24 \mathrm{~h}$, and images were captured under a light microscope using a digital camera system (magnification, $\mathrm{x} 200$ or $\mathrm{x} 40$ ).

Dual-luciferase reporter assay. TargetScan software (version 7.1; www.targetscan.org) was used to predict target genes of miR-25, identifying FBXW7 and DKK3. The 3'UTR of human FBW7 containing the wild type (WT) or mutant (MT) putative miR-25 targeted sequence was cloned into the pMIR-REPORT $^{\text {TM }}$ miRNA Expression Reporter Vector system (Ambion; Thermo Fisher Scientific, Inc.). Subsequently, the 
luciferase reporter plasmid containing either WT-FBXW7 3'UTR or mutant MT-FBXW7 3'UTR was co-transfected with miR-25 mimics or miR-NC mimics into 293T cells (Cell Bank of Type Culture Collection of Chinese Academy of Sciences) using Lipofectamine ${ }^{\circledR} 2000$, according to the manufacturer's protocol. Similarly, the WT and MT 3'UTR of human DKK3 containing the putative miR-25 targeted sequence was cloned into the pMIR-REPORT ${ }^{\mathrm{TM}}$ miRNA Expression Reporter Vector system. Subsequently, the luciferase reporter plasmid containing either WT-DKK3 3'UTR or MT-DKK3 3'UTR was co-transfected with miR-25 mimics or miR-NC mimics into 293T cells using Lipofectamine ${ }^{\circledR} 2000$, according to the manufacturer's protocol. After transfection for $48 \mathrm{~h}$, cultured cells were collected and reporter activities were detected using the Dual-Luciferase Reporter assay system (Promega Corporation), according to the manufacturer's protocol. The activity of firefly luciferase was normalized to the activity of Renilla luciferase.

Western blot analysis. Total protein was extracted from tissues or cells using radioimmunoprecipitation assay buffer (Beyotime Institute of Biotechnology). Cell lysates were centrifuged at $12,000 \mathrm{x}$ g for $5 \mathrm{~min}$ at $4^{\circ} \mathrm{C}$, and the supernatant was collected. Total protein was quantified using the Pierce BCA Protein Assay kit (Thermo Fisher Scientific, Inc.) and $50 \mu \mathrm{g}$ protein/lane was separated via SDS-PAGE on a $12 \%$ gel. The separated proteins were transferred onto PVDF membranes (EMD Millipore) and blocked for $3 \mathrm{~h}$ at room temperature with $5 \%$ skimmed milk in TBS containing Tween-20 (0.5\% TBST). The membranes were incubated with rabbit anti-human FBXW7 primary antibody (1:200; ab105752; Abcam, Cambridge, MA, USA), rabbit anti-human DKK3 primary antibody (1:500; ab187532; Abcam), rabbit anti-human GAPDH primary antibody (1:200; ab9485; Abcam), at room temperature for $3 \mathrm{~h}$. Membranes were washed three times with TBST. Following primary antibody incubation, the membranes were incubated with horseradish peroxidase-conjugated goat anti-rabbit secondary antibody $(1: 5,000 ;$ ab6721; Abcam) at room temperature for $40 \mathrm{~min}$. The protein bands were visualised using enhanced chemiluminescence reagents (ECL; Thermo Fisher Scientific, Inc.). Protein expression was quantified using ImageJ software version 1.46 (National Institutes of Health).

Statistical analysis. Data presented as the mean \pm standard deviation from three independent experiments. All statistical analyses were performed using GraphPad Prism software (ver. 5.0; GraphPad Software, Inc.). Student's t-test (two-tailed) was used to examine differences between two groups. One-way analysis of variance followed by Tukey's post hoc test was used to examine differences among multiple groups. Pearson's correlation analysis was used to assess the relationship of miR-25 expression with FBXW7 or DKK3. Chi-square test was used to assess the association between miR-25 expression and clinicopathological characteristics in patients with glioma. $\mathrm{P}<0.05$ was considered to indicate a statistically significant difference.

\section{Results}

Upregulation of miR-25 is associated with glioma progression. In the current study, miR-25 expression levels were significantly
Table I. Association between miR-25 expression and clinicopathological characteristics in patients with glioma.

\begin{tabular}{lcccc}
\hline Variables & $\begin{array}{c}\text { Number } \\
(\mathrm{n}=60)\end{array}$ & $\begin{array}{c}\text { Low } \\
\text { miR-25 } \\
(\mathrm{n}=31)\end{array}$ & $\begin{array}{c}\text { High } \\
\text { miR-25 } \\
(\mathrm{n}=29)\end{array}$ & P-value \\
\hline Age & & & & 0.208 \\
$\quad<50$ years & 32 & 14 & 18 & \\
$\geq 50$ years & 28 & 17 & 11 & \\
Gender & & 19 & 16 & \\
Male & 35 & 12 & 13 & 0.794 \\
Female & 25 & & & $0.019^{\mathrm{a}}$ \\
WHO stage & & 22 & 11 & \\
I-II & 33 & 9 & 18 & \\
III-IV & 27 & & & \\
\hline
\end{tabular}

${ }^{\mathrm{a}} \mathrm{P}<0.05$. WHO, World Health Organization; miR, microRNA.

higher in glioma tissue compared with normal brain tissue (Fig. 1A). Glioma patients were divided into high and low expression groups, based on the mean value of miR-25 expression in the glioma tissue samples. A significant association of high miR-25 expression with advanced clinical stages was identified (Table I). Furthermore, miR-25 expression was significantly upregulated in several common glioma cell lines compared with NHAs (Fig. 1B).

Knockdown of miR-25 supresses glioma cell proliferation and migration. To further examine the function of miR-25 in glioma, U251 and T98G cells were transfected with miR-25 inhibitor and NC inhibitor. The miR-25 expression levels were significantly reduced in cells transfected with miR-25 inhibitor compared with those transfected with the NC inhibitor (Fig. 2A). In addition, cell proliferation was examined using CCK8 assays, which revealed that knockdown of miR-25 significantly inhibited U251 and T98G cell proliferation compared with the negative control (Fig. 2B and C). Furthermore, cell migration was examined using wound-healing assays, which revealed that knockdown of miR-25 significantly inhibited U251 and T98G cell migration compared with the respective negative control (Fig. 2D and E). These results suggest that miR-25 knockdown inhibits glioma cell proliferation and migration.

FBXW7 and DKK3 are target genes of miR-25 in glioma cells. To further examine the potential underlying regulatory mechanism of miR-25 in glioma progression, TargetScan was used to predict potential target genes of miR-25. FBXW7 and DKK3 were identified as potential functional target genes of miR-25 (Fig. 3A and B). The mRNA expression levels of FBXW7 and DKK3 were significantly downregulated in glioma tissue compared with normal brain tissue (Fig. 3C and D). Furthermore, an inverse correlation between FBXW7 and miR-25 expression and between DKK3 and miR-25 expression was observed in glioma tissues (Fig. 3E and F). 

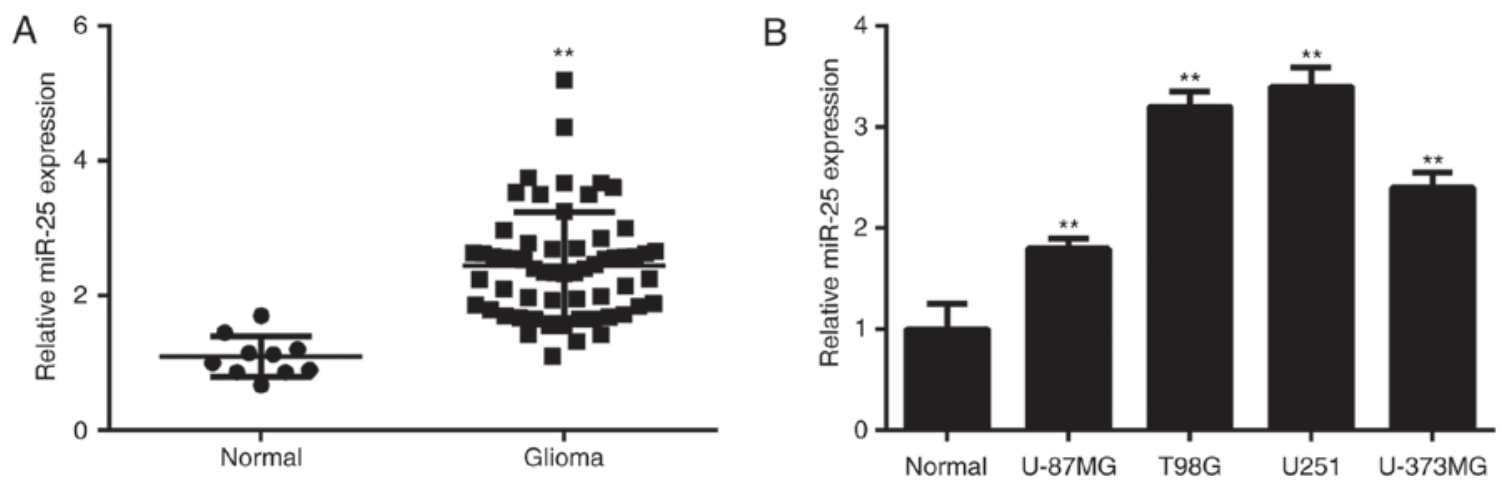

Figure 1. Upregulation of miR-25 is associated with glioma progression. (A) Relative miR-25 expression levels were determined by RT-qPCR in glioma and normal tissue samples. (B) Relative miR-25 expression levels were determined by RT-qPCR in several common glioma cell lines and normal human astrocytes. ${ }^{* *} \mathrm{P}<0.01$ vs. Normal. miR, microRNA; RT-qPCR, reverse transcription-quantitative PCR.

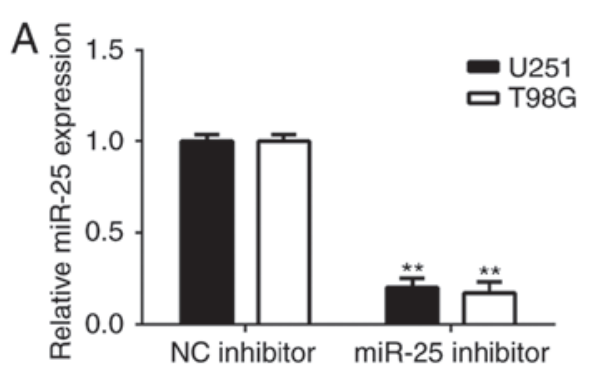

$\mathrm{D}$
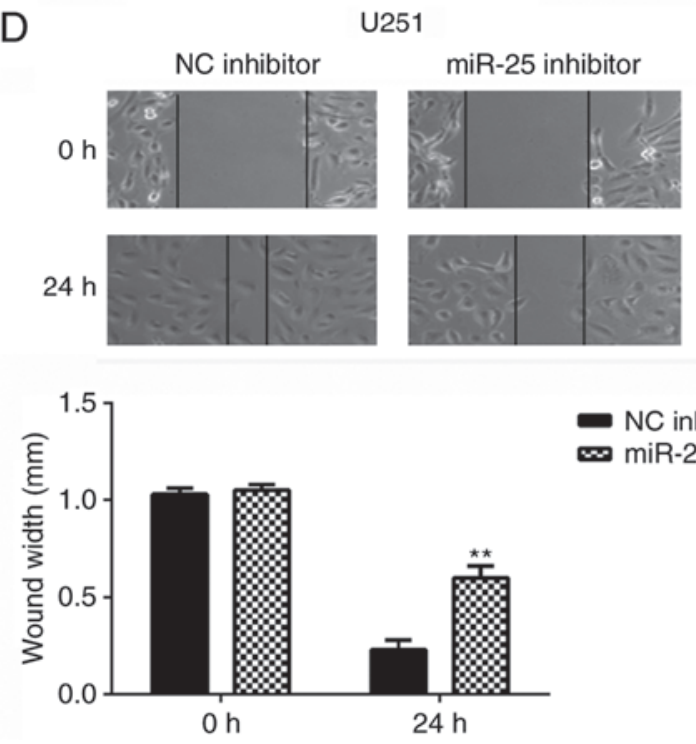
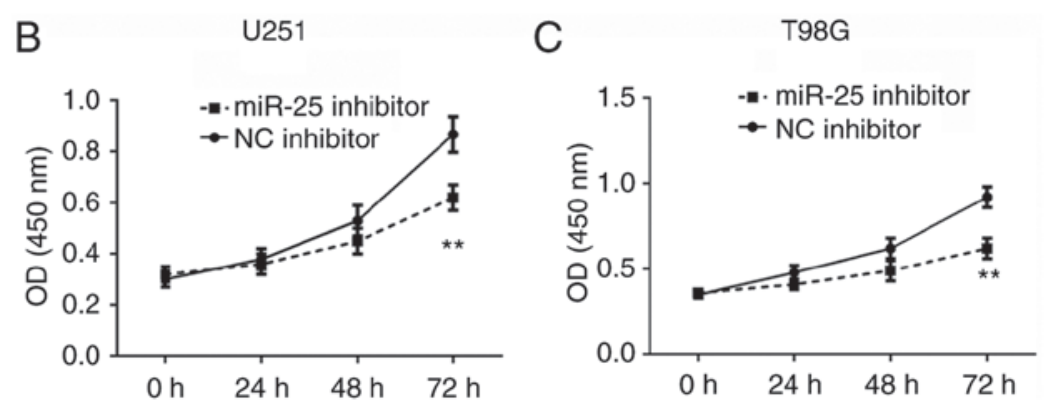

E

T98G
miR-25 inhibitor

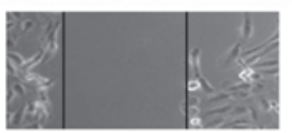

- NC inhibitor $\infty$ miR-25 inhibitor

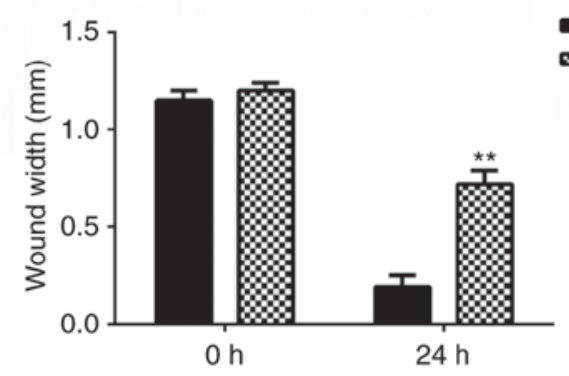

Figure 2. Knockdown of miR-25 suppresses glioma cell proliferation and migration. (A) Relative miR-25 expression levels were determined by RT-qPCR in U251 and T98G cells following transfection with miR-25 inhibitor or NC inhibitor. Cell proliferation was examined by CCK-8 assays in (B) U251 and (C) T98G cells following transfection with miR-25 inhibitor or NC inhibitor. Cell migration was examined using wound-healing assays in (D) U251 (magnification, $\mathrm{x} 40$ ) and (E) T98G cells (magnification, $\mathrm{x} 200$ ) following transfection with miR-25 inhibitor or NC inhibitor. ${ }^{* *} \mathrm{P}<0.01 \mathrm{vs.} \mathrm{NC} \mathrm{inhibitor.} \mathrm{miR,} \mathrm{microRNA;}$ NC, negative control; RT-qPCR, reverse transcription-quantitative PCR; CCK-8, Cell Counting Kit-8.

Dual-luciferase reporter assays were performed to confirm the association between miR-25 and FBXW7, and between miR-25 and DKK3, in glioma cells. Luciferase reporter plasmids containing the WT-DKK3 3'UTR or MT-DKK3 3'UTR, and WT-FBXW7 3'UTR or MT-FBXW7 3'UTR were generated (Fig. 4A and B). The results of the dual-luciferase reporter assays demonstrated that miR-25 overexpression significantly reduced the luciferase activity of the plasmid containing the WT-DKK3 or WT-FBXW7 3'UTR compared with the MT-DKK3 or WT-FBXW7 3'UTR, respectively, which did not affect the luciferase activity. These results confirm that FBXW7 and DKK3 are target genes of miR-25 in glioma cells.

FBXW7 and DKK3 expression is negatively regulated by miR-25 in glioma cells. The effect of miR-25 on FBXW7 and 
A

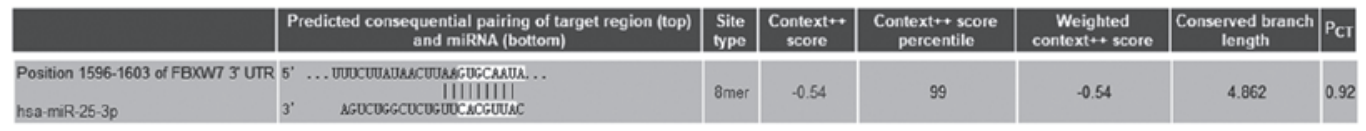

B

\begin{tabular}{|c|c|c|c|c|c|c|c|}
\hline & $\begin{array}{l}\text { Predicted consequential pairing of target region (top) } \\
\text { and miRNA (bottom) }\end{array}$ & $\begin{array}{l}\text { Site } \\
\text { type }\end{array}$ & $\begin{array}{c}\text { Context+c } \\
\text { score }\end{array}$ & $\begin{array}{l}\text { Contex } 1++ \text { score } \\
\text { percentile }\end{array}$ & $\begin{array}{c}\text { Woightod } \\
\text { context }+ \text { score }\end{array}$ & $\begin{array}{c}\text { Consorved branch } \\
\text { length }\end{array}$ & PCT \\
\hline $\begin{array}{l}\text { Position 25-32 of DKK3 } 3^{\prime} \text { UTR } 5^{\prime} \\
\text { hsa-miR-25-3p }\end{array}$ & 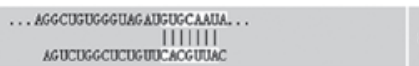 & $8 \mathrm{mer}$ & .0 .52 & 99 & -0.52 & 4.155 & 0.86 \\
\hline
\end{tabular}
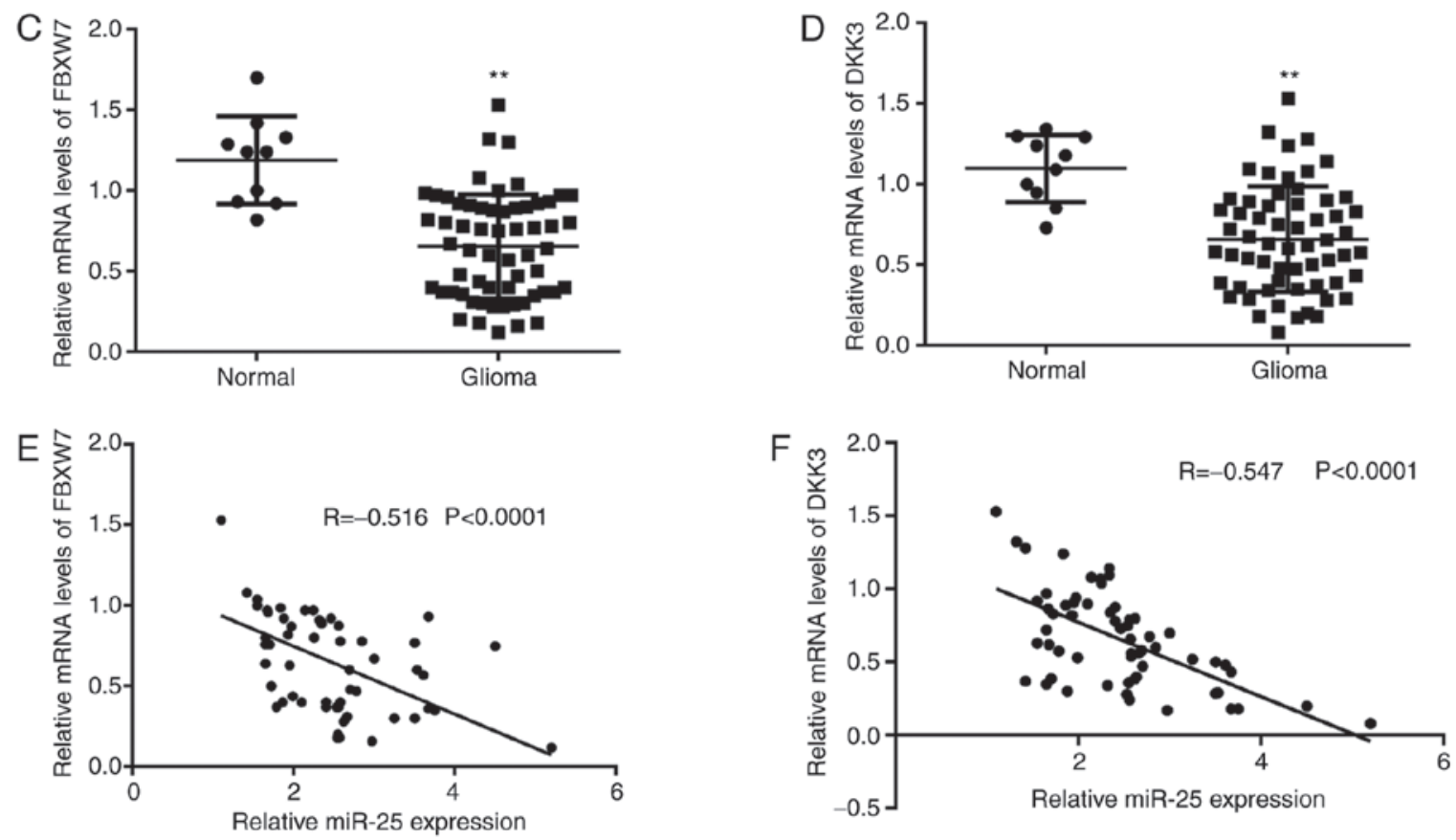

Figure 3. FBXW7 and DKK3 are downregulated in glioma. TargetScan bioinformatics software was used to identify (A) FBXW7 and (B) DKK3 as predicted target genes of miR-25. Relative mRNA expression levels of (C) FBXW7 and (D) DKK3 were determined by RT-qPCR in glioma and normal tissue samples. ${ }^{* *} \mathrm{P}<0.01$ vs. Normal. Relative mRNA expression levels of (E) miR-25 and FBXW7 and (F) miR-25 and DKK3 were inversely correlated in glioma tissues. FBXW7, F-box and WD repeat domain containing 7; DKK3, dickkopf WNT signaling pathway inhibitor 3; miR, microRNA; RT-qPCR, reverse transcriptionquantitative PCR.

A WT DKK3 3'UTR 5'... UAGAUGUGCAAUA...3'<smiles>C#CC#C</smiles>

miR-25 $\quad 3^{\prime} \ldots$...CUGUUCACGUUAC...5'

MT DKK3 3'UTR 5'...UAGAUGUCGUUUA...3'

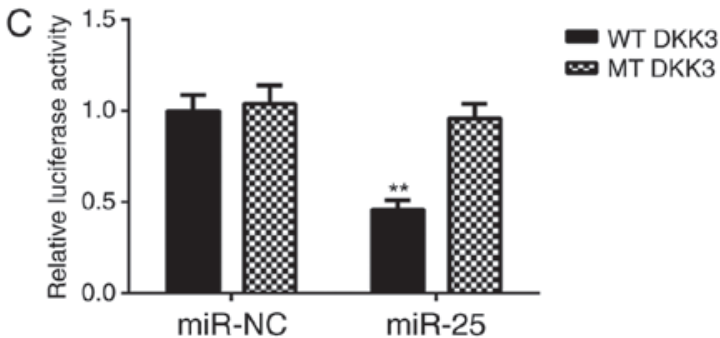

B WT FBXW7 $3^{\prime}$ UTR $\quad 5^{\prime} \ldots$ ACCAGGUGCAAUU...3

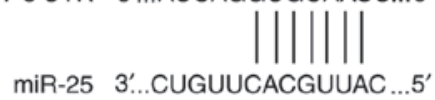

MT FBXW7 3'UTR $55^{\prime} \ldots$...CCAGGUCGUUUU...3'

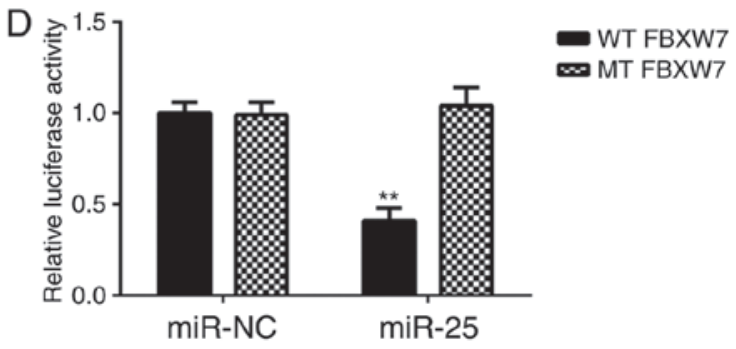

Figure 4. FBXW7 and DKK3 are target genes of miR-25 in glioma cells. Putative WT and MT binding sequences of (A) the 3'-UTR of DKK3 and (B) the 3'-UTR of FBXW7. Dual-luciferase reporter assays were performed following 48-h co-transfection with miR-25 mimics (or miR-NC) and (C) WT-DKK3 (or MT-DKK3) or (D) WT-FBXW7 (or MT-FBXW7) reporter gene plasmid. ${ }^{* *} \mathrm{P}<0.01$ vs. miR-NC. FBXW7, F-box and WD repeat domain containing 7; DKK3, dickkopf WNT signaling pathway inhibitor 3; miR, microRNA; WT, wild-type; MT, mutant; UTR, untranslated region.

DKK3 expression was examined in glioma cells. As shown in Fig. 5A-C, knockdown of miR-25 increased FBXW7 and DKK3 mRNA and protein expression levels in glioma cells. Cells with significantly increased miR-25 expression levels were obtained by transfection with miR-25 mimics compared with negative control (Fig. 5D). The overexpression of miR-25 was demonstrated to decrease FBXW7 and DKK3 expression at the mRNA and protein levels in glioma cells (Fig. 5E-G). 

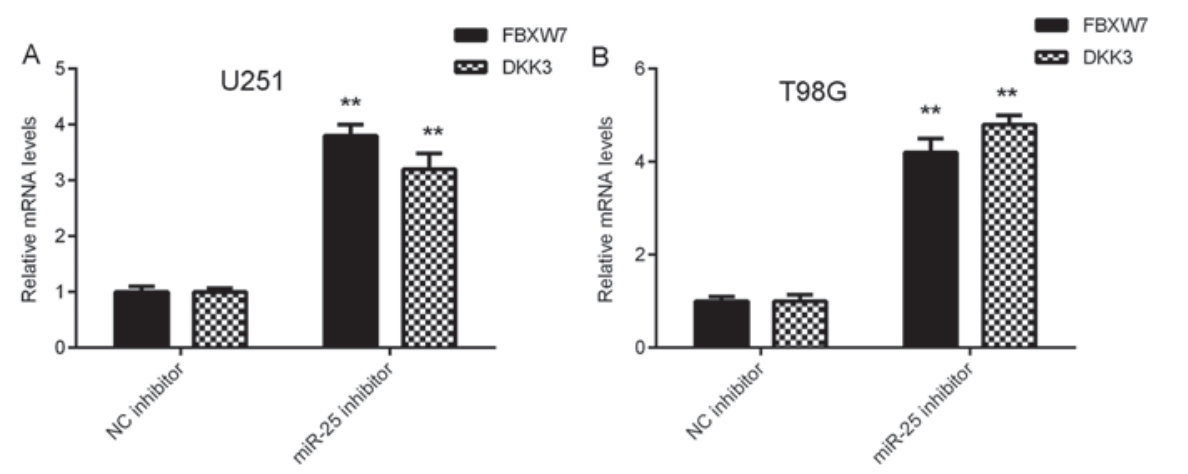

C
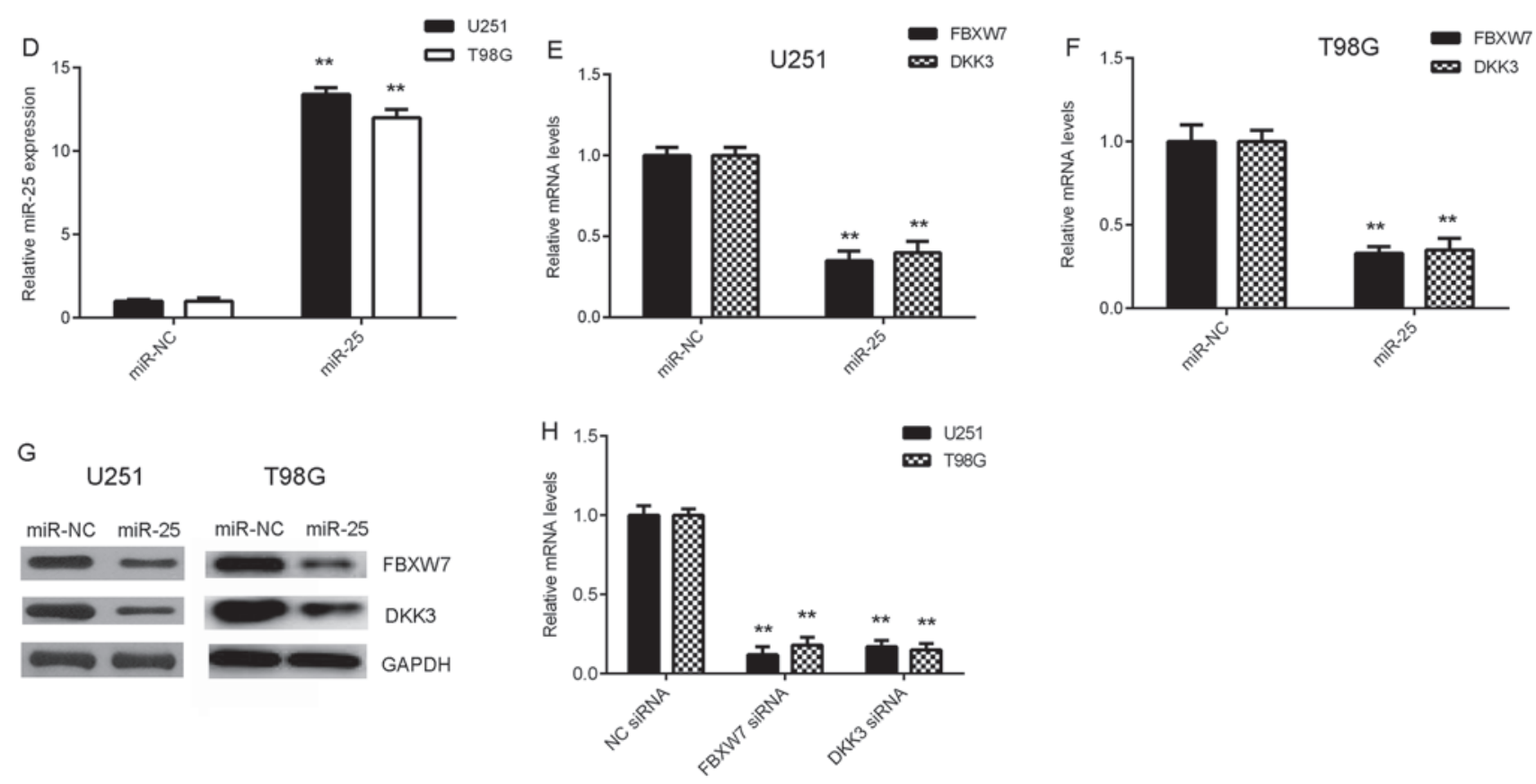

Figure 5. FBXW7 and DKK3 are negatively regulated by miR-25 in glioma cells. Relative FBXW7 and DKK3 mRNA expression levels were determined by RT-qPCR in (A) U251 and (B) T98G cells following transfection with miR-25 inhibitor or NC inhibitor. ${ }^{* *} \mathrm{P}<0.01$ vs. NC inhibitor. (C) FBXW7 and DKK3 protein expression levels were determined by western blotting in U251 and T98G cells following transfection with miR-25 inhibitor or NC inhibitor. (D) Relative miR-25 expression levels were determined by RT-qPCR in U251 and T98G cells following transfection with miR-25 mimics or miR-NC. Relative FBXW7 and DKK3 mRNA expression levels were determined by RT-qPCR in (E) U251 and (F) T98G cells following transfection with miR-25 mimics or miR-NC. ${ }^{* *} \mathrm{P}<0.01$ vs. miR-NC. (G) FBXW7 and DKK3 protein expression levels were determined by western blotting in U251 and T98G cells following transfection with miR-25 mimics or miR-NC. (H) Relative FBXW7 and DKK3 mRNA expression levels were determined by RT-qPCR in U251 and T98G cells following transfection with NC siRNA, FBXW7 siRNA or DKK3 siRNA, respectively. ${ }^{* *} \mathrm{P}<0.01 \mathrm{vs.} \mathrm{NC} \mathrm{siRNA.} \mathrm{FBXW7,} \mathrm{F-box} \mathrm{and} \mathrm{WD} \mathrm{repeat} \mathrm{domain}$ containing 7; DKK3, dickkopf WNT signaling pathway inhibitor 3; miR, microRNA; RT-qPCR, reverse transcription-quantitative PCR; siRNA, small interfering RNA; NC, negative control.

These results suggest that FBXW7 and DKK3 expression is negatively regulated by miR-25 in glioma cells.

In subsequent experiments, glioma cells were transfected with NC siRNA, FBXW7 siRNA or DKK3 siRNA, respectively. RT-qPCR data showed that the mRNA levels of FBXW7 and DKK3 were significantly reduced in the FBXW7 siRNA and DKK3 siRNA groups, when compared with those in the NC siRNA group. Thus, FBXW7 and DKK3 expression levels were successfully downregulated in the U251 and T98G cells (Fig. 5H).

FBXW7 and DKK3 are involved in miR-25-mediated glioma cell proliferation and migration. To investigate whether FBXW7 and DKK3 are involved in miR-25-mediated glioma cell proliferation and migration, U251 and T98G cells were co-transfected with miR-25 inhibitor together with FBXW7
siRNA or DKK3 siRNA. The FBXW7 mRNA and protein expression levels were downregulated in each miR-25 in + siFBXW7 group compared with the respective miR-25 in + siNC group (Fig. 6A and B). In addition, CKK-8 and wound-healing assays demonstrated that glioma cell proliferation and migration were increased in the miR-25 in + siFBXW7 group compared with the respective miR-25 in + siNC group in both cell lines (Fig. 6C-F). Similarly, the relative DKK3 mRNA and protein expression levels were downregulated in the miR-25 in + siDKK3 group compared with the respective miR-25 in + siNC group in both cell lines (Fig. 7A and B). In addition, glioma cell proliferation and migration were increased in the miR-25 in + siDKK3 group compared with the miR-25 in + siNC group (Fig. 7C-F). Taken together, these results suggest that FBXW7 and DKK3 may be involved in miR-25-mediated glioma cell proliferation and migration. 

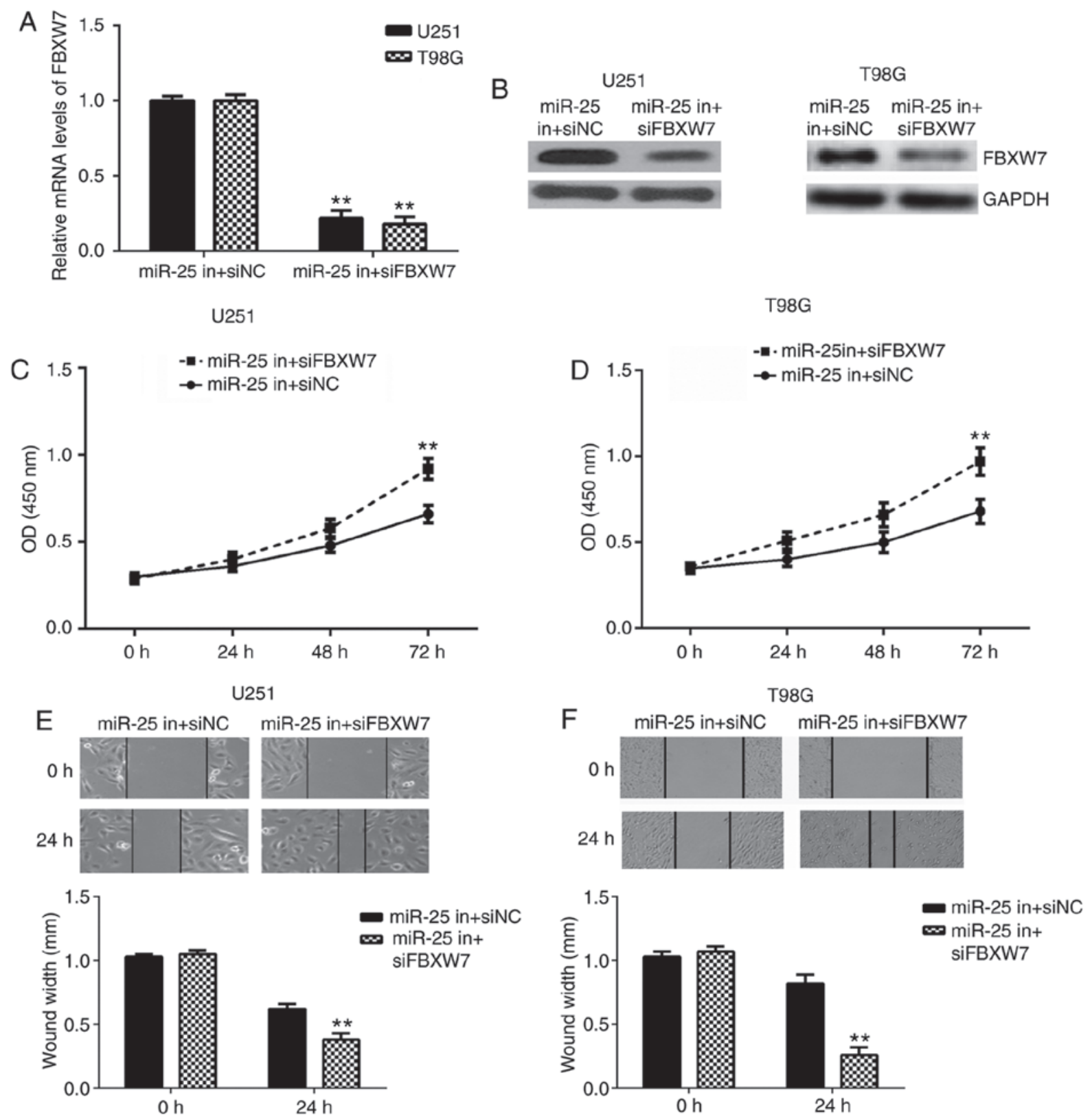

Figure 6. Knockdown of FBXW7 reversed the inhibitory effects of miR-25 downregulation on glioma cell proliferation and migration. The (A) mRNA and (B) protein FBXW7 expression levels were determined by RT-qPCR and western blotting, respectively, in U251 and T98G cells following co-transfection with miR-25 inhibitor together with FBXW7 siRNA or NC siRNA. Cell proliferation was examined by CCK-8 assays in (C) U251 and (D) T98G cells following transfection with miR-25 inhibitor and FBXW7 siRNA or NC siRNA. Cell migration was examined using wound-healing assays in (E) U251 (magnification, $\mathrm{x} 40$ ) and (F) T98G cells (magnification, $\mathrm{x} 200$ ) following transfection with miR-25 inhibitor and FBXW7 siRNA or NC siRNA. ${ }^{* *} \mathrm{P}<0.01 \mathrm{vs.}$ miR-25 in + siNC. FBXW7, F-box and WD repeat domain containing 7; miR, microRNA; RT-qPCR, reverse transcription-quantitative PCR; siRNA, small interfering RNA; NC, negative control; CCK-8, Cell Counting Kit-8.

\section{Discussion}

Since glioma is a malignant brain tumour, it is important to understand the molecular mechanism underlying glioma progression. In the present study, it was observed that miR-25 expression levels were significantly increased in glioma tissue samples and cell lines, and miR-25 upregulation was associated with glioma progression. Knockdown of miR-25 significantly inhibited glioma cell proliferation and migration. FBXW7 and DKK3 were identified as target genes of miR-25. FBXW7 and DKK3 expression levels were significantly downregulated in glioma tissue samples and cells lines, and their expression levels were negatively regulated by miR-25 in glioma cells. Furthermore, knockdown of FBXW7 and DKK3 impaired the miR-25-induced effects on glioma cell proliferation and migration.

A previous study investigated the expression profiles of miRs in four patients with primary gliomas (grade II) that spontaneously progressed to secondary gliomas (grade IV), and demonstrated that miR-25 expression levels increased following progression (24). In the current study, miR-25 was significantly upregulated in glioma tissue compared with normal brain tissue samples as well as in glioma cell lines compared with NHAs. In addition, high miR-25 expression in glioma tissue samples 
A

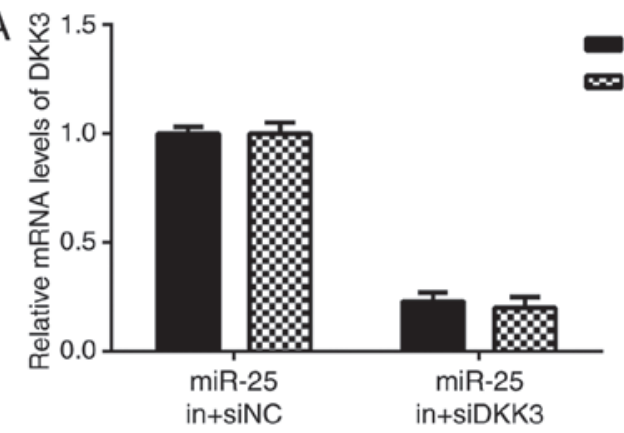

C

U251

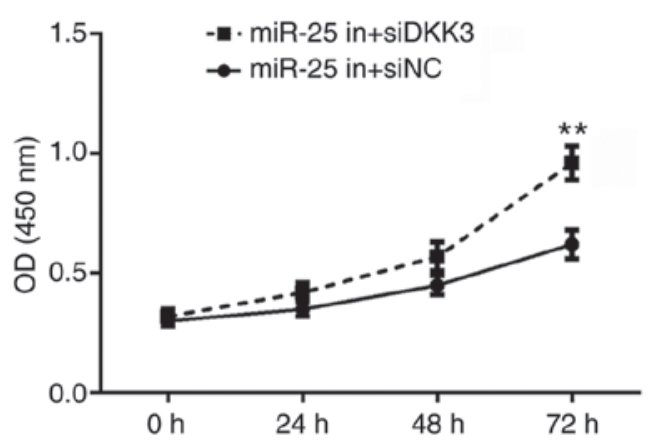

E

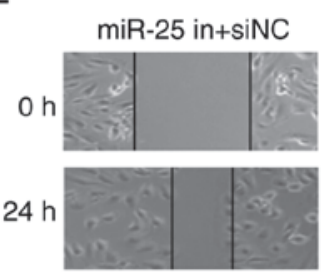

U251
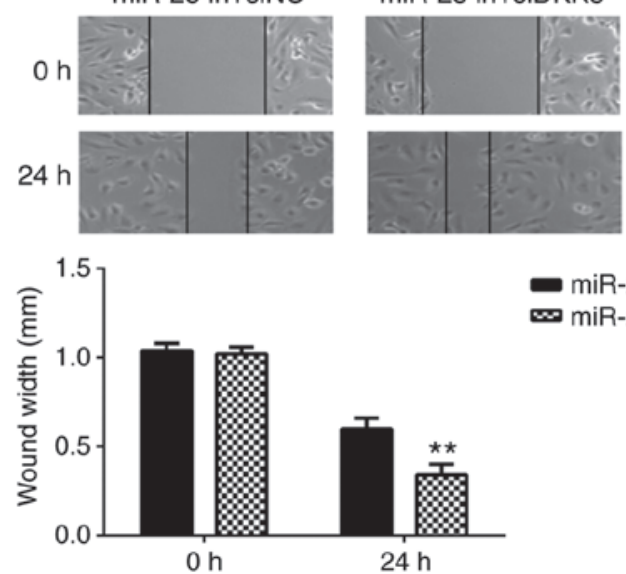

B
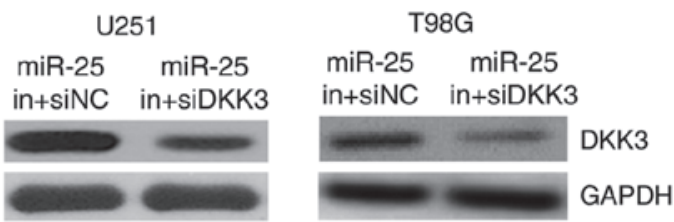

T98G

D

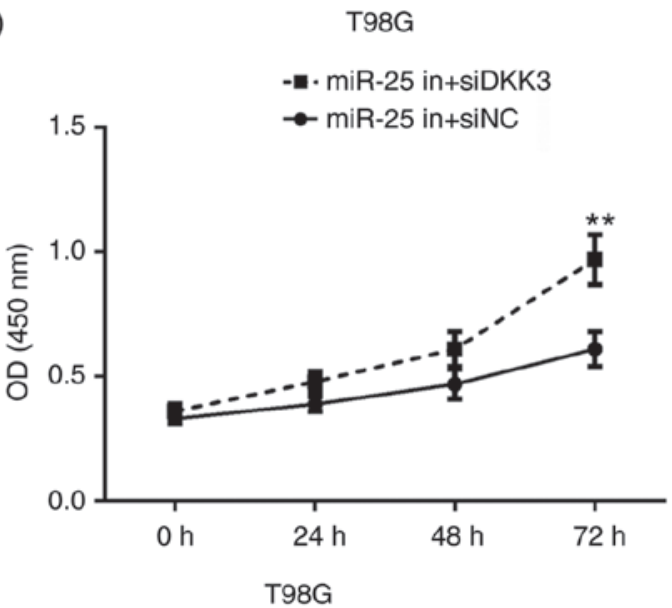

$\mathrm{F}$

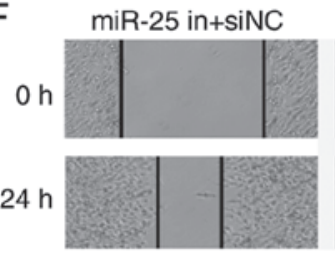

miR-25 in+siDKK3

miR-25 in+siNC $\infty$ miR-25 in+siDKK3

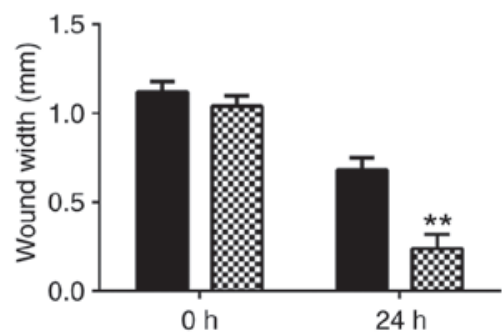

Figure 7. DKK3 knockdown reversed the inhibitory effects of miR-25 downregulation on glioma cell proliferation and migration. The (A) mRNA and (B) protein expression levels of DKK3 were determined by RT-qPCR and western blotting, respectively, in U251 and T98G cells following co-transfection with miR-25 inhibitor together with DKK3 siRNA or NC siRNA. Cell proliferation was examined by CCK-8 assays in (C) U251 and (D) T98G cells following transfection with miR-25 inhibitor and DKK3 siRNA or NC siRNA. Cell migration was examined using wound-healing assays in (E) U251 (magnification, $\mathrm{x} 200$ ) and (F) T98G cells (magnification, $\mathrm{x} 40$ ) following transfection with miR-25 inhibitor and DKK3 siRNA or NC siRNA. ${ }^{* *} \mathrm{P}<0.01$ vs. miR-25 in + siNC. DKK3, dickkopf WNT signaling pathway inhibitor 3; miR, microRNA; RT-qPCR, reverse transcription-quantitative PCR; siRNA, small interfering RNA; NC, negative control; CCK-8, Cell Counting Kit-8.

was associated with advanced clinical stage, which suggests that upregulation of miR-25 may be associated with glioma progression. To further clarify the function of miR-25 in glioma progression, U251 and T98G cell lines were transfected with miR-25 inhibitor. The results demonstrated that the knockdown of miR-25 inhibits glioma cell proliferation and migration.

It is well established that miRs function by regulating the expression of their target genes $(25,26)$. TargetScan bioinformatics software was used in the present study to predict target genes of miR-25. FBXW7 and DKK3 were identified as two potential target genes of miR-25 and so were subsequently examined. Luciferase reporter gene assays confirmed that FBXW7 and DKK3 are direct target genes of miR-25, and their expression was negatively regulated by miR-25 in glioma cell lines. FBXW7 is a well-known tumour suppressor; Hagedorn et al (27) reported that FBXW7 inhibits glioma cell proliferation and is a prognostic marker for survival in patients with glioblastoma, while the loss of FBXW7 serves an important role in glioma malignancy by allowing the accumulation of multiple oncoproteins. Cao et al (28) reported a tumour-suppressive role of the long noncoding RNA metastasis associated lung adenocarcinoma transcript 1 in glioma cells via 
the suppression of miR-155 expression and activation of FBXW7. The current study demonstrated that FBXW7 inhibition attenuated the suppressive effects on glioma cell proliferation and migration induced by miR-25 downregulation, which suggests that FBXW7 may be involved in miR-25-mediated glioma cell proliferation and migration.

DKK3 also functions as a tumour suppressor in glioma; Mizobuchi et al (21) reported that DKK3 was significantly downregulated in glioma tissue compared with normal brain tissue samples, which is consistent with the current study. In addition, overexpression of DKK3 has been reported to inhibit glioma cell proliferation and induce cell apoptosis via the activation of JUN phosphorylation, caspase-9 and caspase-3, and the reduction of $\beta$-catenin (21). Li et al (20) demonstrated that miR-92b increased glioma cell proliferation and inhibited cell apoptosis by targeting DKK3. In the current study, FBXW7 inhibition attenuated the miR-25-induced suppressive effects on glioma cell proliferation and migration, which suggests that DKK3 may also be involved in miR-25-mediated glioma cell proliferation and migration.

An association between miR-25 and FBXW7 has been previously reported in several other types of cancer, including oesophageal squamous cell carcinoma (29), lung cancer (30) and gastric cancer (31). In addition, miR-25 has been found to promote the migration of melanoma cells by targeting DKK3 (32). Thus, the current study increases our understanding of the importance of the miR-25/FBXW7 axis and miR-25/DKK3 axis in human cancer.

To the best of our knowledge, the current study is the first to demonstrate that miR-25 plays a promoting role in glioma cell proliferation and migration, at least in part through directly targeting FBXW7 and DKK3. Therefore, miR-25 may serve as a potential molecular target for the treatment of glioma.

\section{Acknowledgements}

Not applicable.

\section{Funding}

The present study was supported by Scientific Research Project of the Commission of Finance of Hunan province (grant no. 62.2).

\section{Availability of data and materials}

The datasets used and/or analysed during the present study are available from the corresponding author on reasonable request.

\section{Authors' contributions}

CS designed the study and revised the manuscript. GP collected tissue samples, conducted statistical analysis and wrote the manuscript. YL and CY performed all the experiments.

\section{Ethics approval and consent to participate}

The present study was approved by the Ethics Committee of Affiliated Hospital of Xiangya Hospital of Central South
University (Changsha, China). All participants provided written informed consent.

\section{Patient consent for publication}

All participants provided written informed consent.

\section{Competing interests}

The authors declare that they have no competing interests.

\section{References}

1. Siegel RL, Miller KD and Jemal A: Cancer statistics, 2015. CA Cancer J Clin 65: 5-29, 2015.

2. Torre LA, Bray F, Siegel RL, Ferlay J, Lortet-Tieulent J and Jemal A: Global cancer statistics, 2012. CA Cancer J Clin 65: 87-108, 2015.

3. Brower JV, Clark PA, Lyon W and Kuo JS: MicroRNAs in cancer: Glioblastoma and glioblastoma cancer stem cells. Neurochem Int 77: 68-77, 2014

4. Murphy AM and Rabkin SD: Current status of gene therapy for brain tumors. Transl Res 161: 339-354, 2013.

5. Zhang Z,Li X, Xiao Q and Wang Z: MiR-574-5p mediates the cell cycle and apoptosis in thyroid cancer cells via Wnt/beta-catenin signaling by repressing the expression of Quaking proteins. Oncol Lett 15: 5841-5848, 2018.

6. Luo T, Yan Y, He Q, Ma X and Wang W: miR-328-5p inhibits MDA-MB-231 breast cancer cell proliferation by targeting RAGE. Oncol Rep 39: 2906-2914, 2018.

7. Li Z, Guo J, Ma Y, Lin Z and Zhang L: Oncogenic role of MicroRNA-30b-5p in glioblastoma through targeting proline-rich transmembrane protein 2. Oncol Res 26: 219-230, 2018.

8. Xu R, Zhu X, Chen F, Huang C, Ai K, Wu H, Zhang L and Zhao X: LncRNA XIST/miR-200c regulates the stemness properties and tumourigenicity of human bladder cancer stem cell-like cells. Cancer Cell Int 18: 41, 2018.

9. Zhu Y, Zhao H, Rao M and Xu S: MicroRNA-365 inhibits proliferation, migration and invasion of glioma by targeting PIK3R3. Oncol Rep 37: 2185-2192, 2017.

10. Zhang T, Ma G, Zhang Y, Huo H and Zhao Y: miR-599 inhibits proliferation and invasion of glioma by targeting periostin. Biotechnol Lett 39: 1325-1333, 2017.

11. Jiang L, Wang C, Lei F, Zhang L, Zhang X, Liu A, Wu G, Zhu J and Song L: miR-93 promotes cell proliferation in gliomas through activation of PI3K/Akt signaling pathway. Oncotarget 6: 8286-8299, 2015.

12. Chen H, Pan H, Qian Y, Zhou W and Liu X: MiR-25-3p promotes the proliferation of triple negative breast cancer by targeting BTG2. Mol Cancer 17: 4, 2018.

13. Casadei L, Calore F, Creighton CJ, Guescini M, Batte K, Iwenofu OH, Zewdu A, Braggio DA, Bill KL, Fadda P, et al: Exosome-derived miR-25-3p and miR-92a-3p stimulate liposarcoma progression. Cancer Res 77: 3846-3856, 2017.

14. Zhang J, Gong X, Tian K, Chen D, Sun J, Wang G and Guo M: miR-25 promotes glioma cell proliferation by targeting CDKN1C. Biomed Pharmacother 71: 7-14, 2015.

15. Peng G, Yuan X, Yuan J, Liu Q, Dai M, Shen C, Ma J, Liao Y and Jiang W: miR-25 promotes glioblastoma cell proliferation and invasion by directly targeting NEFL. Mol Cell Biochem 409: 103-111, 2015.

16. Cao J, Ge MH and Ling ZQ: Fbxw7 tumor suppressor: A vital regulator contributes to human tumorigenesis. Medicine (Baltimore) 95: e2496, 2016.

17. Zhou C, Shen L, Mao L, Wang B, Li Y and Yu H: miR-92a is upregulated in cervical cancer and promotes cell proliferation and invasion by targeting FBXW7. Biochem Biophys Res Commun 458: 63-69, 2015.

18. Lin J, Ji A, Qiu G, Feng H, Li J, Li S, Zou Y, Cui Y, Song C, He H and Lu Y: FBW7 is associated with prognosis, inhibits malignancies and enhances temozolomide sensitivity in glioblastoma cells. Cancer Sci 109: 1001-1011, 2018.

19. Lee EJ, Jo M, Rho SB, Park K, Yoo YN, Park J, Chae M, Zhang W and Lee JH: Dkk3, downregulated in cervical cancer, functions as a negative regulator of beta-catenin. Int J Cancer 124: 287-297, 2009. 
20. Li Q, Shen K, Zhao Y, Ma C, Liu J and Ma J: MiR-92b inhibitor promoted glioma cell apoptosis via targeting DKK3 and blocking the Wnt/beta-catenin signaling pathway. J Transl Med 11: 302, 2013.

21. Mizobuchi Y, Matsuzaki K, Kuwayama K, Kitazato K, Mure H, Kageji T and Nagahiro S: REIC/Dkk-3 induces cell death in human malignant glioma. Neuro Oncol 10: 244-253, 2008.

22. Pisapia DJ: The updated world health organization glioma classification: Cellular and molecular origins of adult infiltrating gliomas. Arch Pathol Lab Med 141: 1633-1645, 2017.

23. Livak KJ and Schmittgen TD: Analysis of relative gene expression data using real-time quantitative PCR and the 2(-Delta Delta C(T)) method. Methods 25: 402-408, 2001.

24. Malzkorn B, Wolter M, Liesenberg F, Grzendowski M, Stühler K, Meyer HE and Reifenberger G: Identification and functional characterization of microRNAs involved in the malignant progression of gliomas. Brain Pathol 20: 539-550, 2010.

25. Xiao F, Li Y, Wan Y and Xue M: MircroRNA-139 sensitizes ovarian cancer cell to cisplatin-based chemotherapy through regulation of ATP7A/B. Cancer Chemother Pharmacol 81: 935-947, 2018.

26. Ruan L, Chen J, Ruan L, Yang T and Wang P: MicroRNA-186 suppresses lung cancer progression by targeting SIRT6. Cancer Biomark 21: 415-423, 2018.
27. Hagedorn M, Delugin M, Abraldes I, Allain N, Belaud-Rotureau MA, Turmo M, Prigent C, Loiseau H, Bikfalvi A and Javerzat S: FBXW7/hCDC4 controls glioma cell proliferation in vitro and is a prognostic marker for survival in glioblastoma patients. Cell Div 2: 9, 2007.

28. Cao S, Wang Y, Li J, Lv M, Niu H and Tian Y: Tumor-suppressive function of long noncoding RNA MALAT1 in glioma cells by suppressing miR-155 expression and activating FBXW7 function. Am J Cancer Res 6: 2561-2574, 2016.

29. Hua Y, Zhao K, Tao G, Dai C and Su Y: miR-25 promotes metastasis via targeting FBXW7 in esophageal squamous cell carcinoma. Oncol Rep 38: 3030-3038, 2017.

30. Xiang J, Hang JB, Che JM and Li HC: MiR-25 is up-regulated in non-small cell lung cancer and promotes cell proliferation and motility by targeting FBXW7. Int J Clin Exp Pathol 8: 9147-9153, 2015.

31. Gong J, Cui Z, Li L, Ma Q, Wang Q, Gao Y and Sun H: MicroRNA-25 promotes gastric cancer proliferation, invasion, and migration by directly targeting F-box and WD-40 domain protein 7, FBXW7. Tumour Biol 36: 7831-7840, 2015.

32. Huo J, Zhang Y, Li R, Wang Y, Wu J and Zhang D: Upregulated MicroRNA-25 mediates the migration of melanoma cells by targeting DKK3 through the WNT/beta-Catenin Pathway. Int J Mol Sci 17: E1124, 2016. 